\title{
Pharmaceutical care issues encountered by post-menopausal osteoporotic women prescribed bisphosphonates
}

\author{
P. S. M. Lai*† PhD, S. S. Chua $\uparrow$ PhD and S. P. Chan $\ddagger$ MBBS, MRCP (Edin) \\ *Department of Primary Care Medicine, Faculty of Medicine, University of Malaya, $\dagger$ Department of Pharmacy, Faculty of Medicine, University of Malaya \\ and $\ddagger$ Department of Medicine, Faculty of Medicine, University of Malaya, Kuala Lumpur, Malaysia
}

Received 1 February 2011, Accepted 22 November 2011

Keywords: bisphosphonates, Malaysia, osteoporosis, pharmaceutical care issues, pharmacist intervention, post-menopausal

\section{SUMMARY}

What is known and Objective: Pharmacists have been involved in providing comprehensive interventions to osteoporosis patients, but pharmaceutical care issues (PCIs) encountered during such interventions have not been well documented. Therefore, the aim of this study was to document PCIs encountered by post-menopausal osteoporotic women prescribed bisphosphonates.

Methods: A randomized controlled study was conducted from September 2005-February 2009 in the University Malaya Medical Centre, Malaysia. This main intervention study measured the effects of pharmaceutical care on medication adherence, persistence, quality of life, knowledge and patient satisfaction. However, this manuscript is part of the main intervention study and focuses only on the PCIs encountered. Inclusion criteria: post-menopausal women diagnosed with osteoporosis ( $\mathrm{T}$-score $\leq-2 \cdot 5 /$ low-trauma fracture) and prescribed weekly alendronate/risedronate. Exclusion criteria: those with metabolic bone disease and could not communicate in English. The PCIs identified were collected via personal interviews or telephone calls, and each participant was followedup for a period of 2 years. All PCIs were discussed with and confirmed by a physician.

Results and Discussion: Of the 198 participants recruited, 64 (32.3\%) experienced adverse effects because of bisphosphonates whereas one participant $(0.5 \%)$ refused to start bisphosphonates because of fear of adverse effects. Most adverse effects [50 (74.6\%)] were reported during the first 3 months of therapy with gastrointestinal problems being the main issue [23 (11.6\%)]. Of the nine participants (4.5\%) who discontinued bisphosphonates, only three agreed to take another medication. From the 97 PCIs identified, 77 issues could be classified as drug-related problems [according to the Pharmaceutical Care Network Europe Classification v6.2]. There were 87 causes, 178 interventions and 77 outcomes. The main problem and cause of the PCIs encountered were adverse drug events $(83.1 \%$ and $74.7 \%$ respectively), whereas the main intervention provided was patient counselling $(\mathbf{4 1 . 0 \% )}$. All problems were solved (98.5\%) except for one because of the lack of cooperation of a patient. After 2 years, 36 participants (20\%) were no longer per-

Correspondence: Dr P. S. M. Lai, Department of Primary Care Medicine, Faculty of Medicine, University of Malaya, 50603 Kuala Lumpur, Malaysia. Tel.: +60379492306; fax: +60379577941; e-mail: plai@ummc.edu.my

Submitted as an abstract to the 23rd Federation of Asian Pharmaceutical Association Congress, Taipei, Taiwan, 5-8 November 2010. sistent with therapy, $19(10.6 \%)$ did not have clinic follow-up and $53(26.8 \%)$ did not have a bone mineral density scan.

What is new and Conclusion: The main PCIs identified were related to the use of bisphosphonates and its adverse effects. The study showed that the presence of a clinical pharmacist has enabled patients to voice their medication-related issues and to allow appropriate recommendations and actions to be taken to resolve these issues.

\section{WHAT IS KNOWN AND OBJECTIVE}

Osteoporosis is a 'disease characterized by low bone mass and micro-architectural deterioration of bone tissue, enhanced bone fragility and an increase in fracture risk'. ${ }^{1}$ The loss of bone occurs 'silently' and progressively and often there is no symptom until the first fracture happens. ${ }^{2}$ As the disease progresses, symptoms may include back pain, fractures, loss of height, skeletal deformity, neck strain, mid-abdominal pain, alterations in bowel functions and rarely, lung disease. ${ }^{3}$

Osteoporosis has become a major public health concern worldwide. Although osteoporosis is more prevalent in women, it can also affect men. ${ }^{4}$ This disease can occur at any age, and in any racial or ethnic group. However, it is more common in post-menopausal women, especially Asians or Caucasians. ${ }^{5}$ Because of the enormous medical and economic impact of osteoporosis, measures must be taken to increase awareness, detection, prevention and treatment of this disease.

Pharmacological interventions for the prevention and treatment of osteoporosis include vitamin and mineral supplementation, bisphosphonates, hormone replacement therapy, selective oestrogen receptor modulators, calcitonin, teriparatide and strontium. However, medications are only safe and effective if used correctly.

Pharmacists are in a strategic position to provide pharmaceutical care, which is defined as the responsible provision of drug therapy for the purpose of achieving definite outcomes that improve quality of life (QOL). ${ }^{6}$ Pharmacists should counsel patients on how best to take their medications, which is especially important for bisphosphonates, as they need to be taken in a specific manner and for at least a year to be effective in maintaining bone mineral density (BMD) and to protect against fracture. ${ }^{7}$ A review of the literature found that pharmacists have been involved in providing comprehensive interventions on osteoporosis patients through screening, early detection of osteoporosis, ${ }^{8-15}$ monitoring of drug therapy ${ }^{8,16}$ and patient education, ${ }^{12,17,18}$ but such interventions and the pharmaceutical care issues (PCIs) encountered have not been well documented. 
Therefore, the aim of this study was to document PCIs encountered by post-menopausal osteoporotic women prescribed bisphosphonates. Clinical and health outcomes (such as change in BMD, falls and fractures) after 2 years were also determined.

\section{METHODS}

A randomized controlled trial (RCT) was conducted from September 2005 to February 2009 in the University Malaya Medical Centre (UMMC), Malaysia. This main intervention study measured the effects of pharmaceutical care on medication adherence, persistence, QOL, knowledge and patient satisfaction. Three methods were used to assess medication adherence: directreporting (by asking the participant, 'How many doses of alendronate/risedronate did you miss since the last time we met?'), pill count (by counting the number of bisphosphonate tablets left at each visit) and self-recording (by asking participants to record the date they took their bisphosphonates). ${ }^{19}$ These methods were validated in a preliminary study on 227 post-menopausal osteoporotic women prescribed once-weekly bisphosphonates. Medication adherence was assessed using the self-recording method as the exact day when the participant took her weekly medication could be determined. Details on how adherence was measured have previously been published. ${ }^{19}$ The provision of pharmaceutical care improved medication adherence but not persistence. ${ }^{19}$ QOL, knowledge and patient satisfaction were also higher in participants provided with pharmaceutical care. ${ }^{20,21}$ This manuscript is part of the RCT but focuses only on the PCIs encountered. Approval from the Medical Ethics Committee of UMMC was obtained before commencement of the study. All participants provided informed written consent.

\section{Participants}

Inclusion criteria: Post-menopausal women who had just been diagnosed with osteoporosis (BMD T-score $\leq-2 \cdot 5 /$ low-trauma fracture), never been on any osteoporosis therapy within the past 6 months and have just been prescribed once-weekly alendronate $\left(\right.$ Fosamax $^{\circledR}$, Merck Sharp \& Dohme Corp., Pavia, Italy) or risedronate (Actonel ${ }^{\circledR}$, OSG Norwich Pharmaceuticals Inc., North Norwich, NY, USA). Exclusion criteria: Patients with metabolic bone disease or other medical conditions or treatment likely to affect bone metabolism, history of chronic renal, hepatic or gastrointestinal (GI) disease or traumatic lumbar compression fracture.

\section{Sample size}

Because this manuscript is part of an intervention study, the sample size used was based on the main outcome measure of the intervention study, which was medication adherence. To detect a mean difference of $5 \%$ with a pooled standard deviation of $10 \%$ between the control and intervention group, with $80 \%$ power of detection and $\alpha=0 \cdot 05$, a sample size of at least 64 was required in each group. ${ }^{22}$ Assuming a $20 \%$ loss to follow-up, ${ }^{23}$ the total number of participants required was at least 77 in each arm.

\section{Outcome measure}

Outcome measure of this part of the study was PCIs encountered by osteoporosis patients. Clinical outcomes of participants such as change in BMD, falls and fractures after 2 years were also assessed as these have an impact on the long-term management of osteoporosis patients.

\section{Procedure}

Participants were randomly allocated to the control or intervention group using the random digits table. ${ }^{24}$ At baseline, demographic data of participants were collected and bisphosphonate was started. Osteoporosis medications were dispensed to all participants, with an explanation on how to take the medications.

Intervention was provided by the research pharmacist at months $0,3,6$ and 12. Intervention participants received a 'counselling package' which consisted of an explanation on osteoporosis, risk factors, lifestyle modifications, goals of osteoporosis therapy, adverse effects and the importance of medication adherence. A medication review was also conducted. Control participants did not receive any additional counselling. However, at month 12, control participants were provided the same 'counselling package'. Both control and intervention participants were then provided with standard pharmacy service for a year before they were interviewed again concerning their clinical status and also any PCI encountered.

The PCIs identified were collected via personal interviews or telephone calls at months 0, 1, 2, 3, 4, 5, 6, 9, 12 and 24. Personal interviews were conducted at each intervention visit (months 0 , 3, 6 and 12), although participants were contacted by telephone calls for the other months. Each participant was followed-up for a period of 2 years. The differences in the number and type of PCIs encountered between the control and intervention group were not assessed in the present study as more PCIs may be reported by intervention participants (because they were in constant contact with the pharmacist and were more likely to discuss their problems) than the control group. Therefore, PCIs encountered by both control and intervention participants were combined in this report. All PCIs were validated with another physician, and endoscopy was conducted if required. PCIs identified were then classified according to the Pharmaceutical Care Network Europe (PCNE) Classification v6.2. ${ }^{25}$

\section{Instruments used}

A pharmaceutical care plan was used to document all the PCIs. This care plan was developed in the English language as English is understood by most pharmacists practicing in Malaysia.

\section{Statistical analyses}

All data were entered into the Statistical Package for Social Sciences (SPSS) version 15 (Chicago, Illinois, USA). Continuous data were expressed as mean \pm standard deviation (SD). Categorical variables were expressed as absolute (number) and relative frequencies (percentage).

\section{RESULTS AND DISCUSSION}

A total of 198 participants were recruited in this study. Participants' demographic data are shown in Table 1.

\section{Pharmaceutical care issues}

A total of 97 PCIs were reported (Table 2). The main PCIs encountered were attributed to the use of bisphosphonates. A 
Table 1. Demographic data of participants at baseline

\begin{tabular}{lc}
\hline & No. of participants \\
Characteristics & $\%(n=198)$ \\
\hline & \\
Mean age \pm SD (years) [range] & $66 \cdot 1 \pm 9 \cdot 3[42-94]$ \\
Ethnicity & \\
Malay & \\
Chinese & $31(15 \cdot 7)$ \\
Indian \& others ${ }^{\text {a }}$ & $114(57 \cdot 6)$ \\
BMI [mean \pm SD] & $53(26 \cdot 8)$ \\
& $23 \cdot 8 \pm 4 \cdot 4$ \\
BMI range & \\
$<18 \cdot 5$ (underweight) & \\
18.5-24.9 (normal) & $20(10 \cdot 1)$ \\
$25 \cdot 0-29 \cdot 9$ (overweight) & $107(54 \cdot 0)$ \\
$\geq 30$ (obese) & $54(27 \cdot 3)$ \\
& $17(8 \cdot 6)$ \\
Level of education & \\
No formal education & \\
Primary education & $16(8 \cdot 1)$ \\
Secondary education & $43(21 \cdot 7)$ \\
Diploma/tertiary/postgraduate & $82(41 \cdot 4)$ \\
Had a previous fall or fracture & $57(28 \cdot 8)$ \\
No. of years menopausal [mean \pm SD] & $101(51 \cdot 0)$ \\
Family history of osteoporosis & $17 \cdot 4 \pm 9 \cdot 5$ \\
\hline
\end{tabular}

SD, Standard deviation.

aOne participant who was classified under 'others' was included under the Indian group.

total of 64 participants (32.3\%) experienced adverse effects, which is slightly less than the $47.8 \%$ reported by another study, ${ }^{26}$ whereas one participant $(0.5 \%)$ refused to start taking bisphosphonates because of fear of adverse effects. Most [50 $(74.6 \%)]$ adverse effects were reported during the first 3 months of therapy and were mainly because of the 'first dose effect', with GI problems being the main issue [23 (11.6\%)], followed by musculoskeletal pain, headache and fever. Five (2.5\%) participants were sent for endoscopy and all were confirmed to have upper GI ulcers. Some adverse effects were so severe that nine participants $(4.5 \%)$ discontinued their bisphosphonates, which is similar to other studies. ${ }^{27-30}$ Of these nine participants, only three agreed to take another medication for their osteoporosis.

Despite having experienced adverse effects, most participants persisted with their bisphosphonates after reassurance by the pharmacist that these effects were transient. Participants who had GI effects were counselled again as to the correct method of taking bisphosphonates, whereas participants who experienced musculoskeletal pain and fever were advised to take paracetamol to relieve these symptoms. Ultimately, after 2-3 weeks of therapy, these adverse effects eventually disappeared and participants were happy to continue their medication. This indicates that pharmacists play an essential role in counselling patients on the correct method of taking bisphosphonates and recommending appropriate actions to relieve these symptoms.

Although patients should be warned of common adverse effects of medications, there is also a distinct risk that such information might be misunderstood, leading to unjustifiable fear of adverse effects. Subsequently, the patient may refuse to take any medication ${ }^{31}$ as observed in one participant. Although the pharmacist spent additional time to educate the patient about her health problem and the benefits of therapy, the participant still refused to start her osteoporosis medication. However, a year later, she started taking the once-weekly bisphosphonates when her BMD scan showed further deterioration.

Other PCIs documented were: no effect of drug treatment (2), effect of drug treatment not optimal (3), unnecessary drug treatment (7), therapy recommendation (4) and lifestyle changes (16) (Table 2).

According to the PCNE classification (v6.2), therapy recommendations and lifestyle changes were not considered as drugrelated problems (DRPs), and one problem can have more than one cause, which can lead to more than one intervention, but only one outcome. Of the 97 PCIs identified, 77 issues were classified as DRPs, which were associated with 87 causes, 178 interventions and 77 outcomes (Table 2). The main problem and cause of the PCIs encountered were adverse drug events (83.1\% and $74.7 \%$, respectively), whereas the main intervention provided was patient counselling $(41.0 \%)$. All problems were solved (98.5\%) except for one because of the lack of cooperation of a patient. All the DRPs were discussed with and confirmed by a physician. Some of the findings could not be classified appropriately such as 'the patient's fear of starting therapy' and 'the cause of adverse effects', hence 'P4-1: Patient dissatisfied with therapy despite optimal clinical and economic treatment outcomes' and 'C $8 \cdot 1$ : Other cause (adverse effect of drug)' were considered as the closest options. Another limitation of the PCNE v6.2 was that therapy recommendations and lifestyle changes were not considered as DRPs.

Of the 97 PCIs reported, a total of 91 (93.8\%) recommendations were provided by the clinical pharmacist and accepted by the prescriber. Six PCIs were referred directly to the prescriber for further action. Similarly, in other studies, $89.4 \%{ }^{32}$ and $95.9 \%$ 33 of pharmacists' recommendations made to physicians were accepted. However, one study showed a low acceptance rate $(39 \%),{ }^{34}$ which may be due to the indirect contact between the pharmacist and the physician.

During the 2-year study period, the participants asked a total of 324 questions. The most common questions asked were the side effects of bisphosphonates [69 (34.8\%) participants], duration of bisphosphonate therapy [48 (24.2\%) participants] and how to take bisphosphonates [43 (21.7\%) participants]. Participants in the present study appeared confused about the duration they had to be on bisphosphonates. Ten (5.1\%) participants believed that they had to remain on therapy for only 6 months, whereas $38(19 \cdot 2 \%)$ participants believed that their osteoporosis medications had to be taken life-long. This calls for more effective communication between patients and their healthcare providers to ensure better understanding of osteoporosis therapy.

\section{Outcomes of participants after two years}

Out of 198 participants, $16(8 \cdot 1 \%)$ could not be contacted due to a change of telephone numbers, and $2(1.0 \%)$ passed away because of reasons unrelated to osteoporosis. Therefore, only 180 participants (90.9\%) were included in subsequent analyses. Thirty-six (20\%) participants discontinued their bisphosphonates because of various reasons (Fig. 1). In addition, 19 (10.6\%) participants defaulted clinic follow-ups because of the following reasons: $5(2.5 \%)$ were too busy, $4(2.0 \%)$ had too many other clinic appointments, $4(2.0 \%)$ felt that there was no necessity for follow-up as they were no longer taking any osteoporosis medications, $2(1.0 \%)$ felt that they were feeling better, 2 (1.0\%) had 

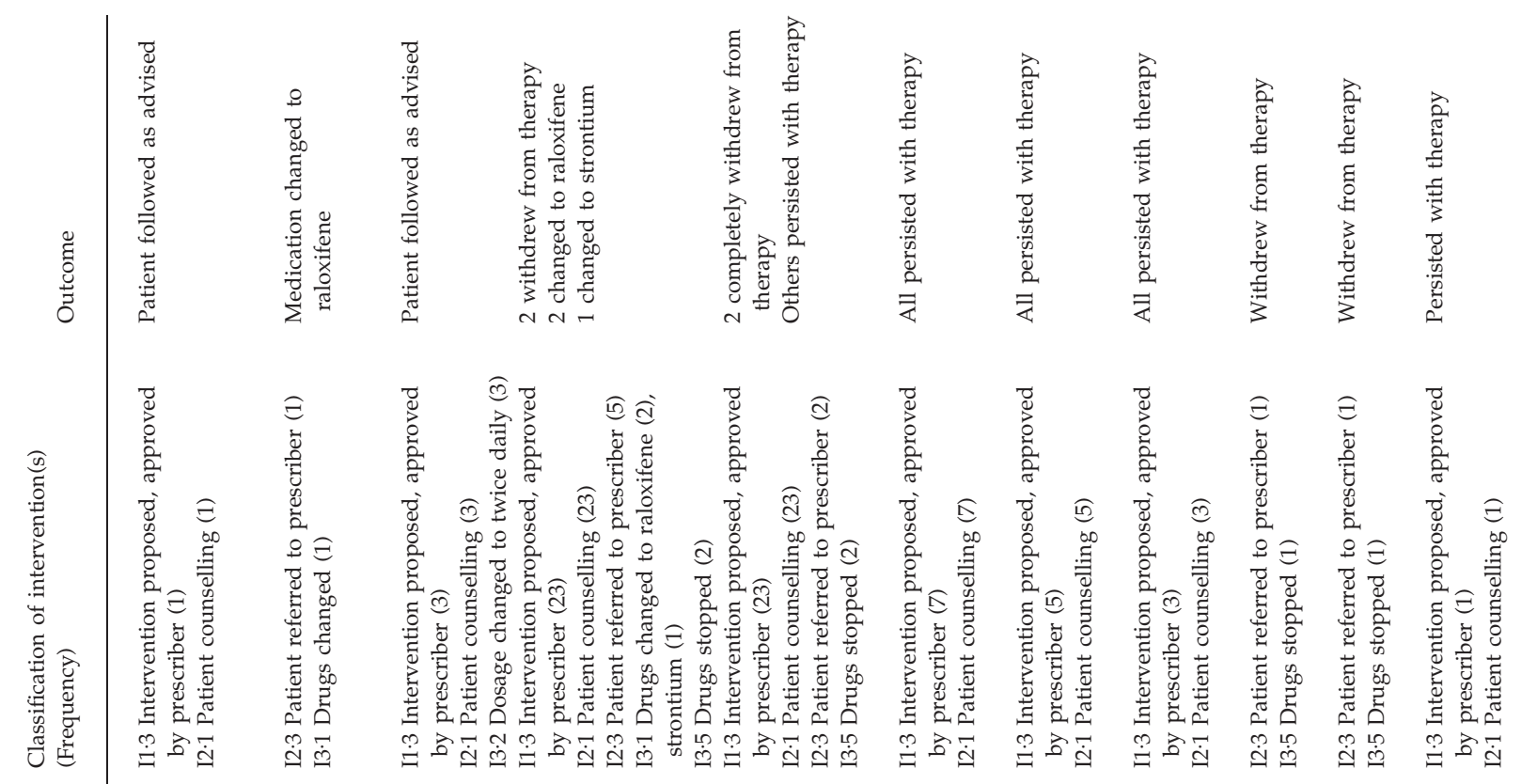

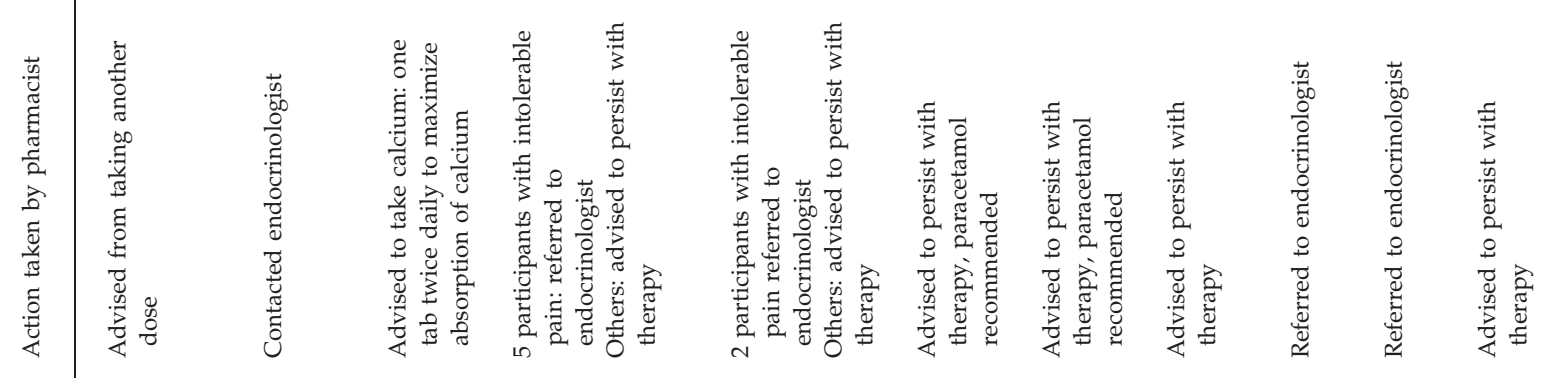

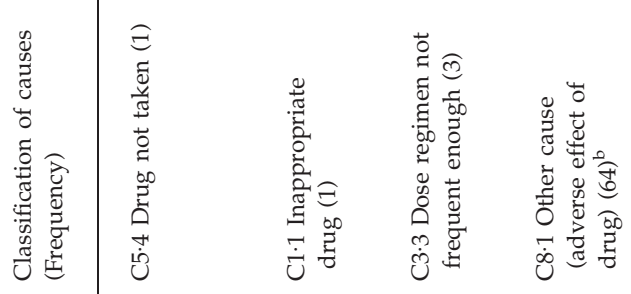




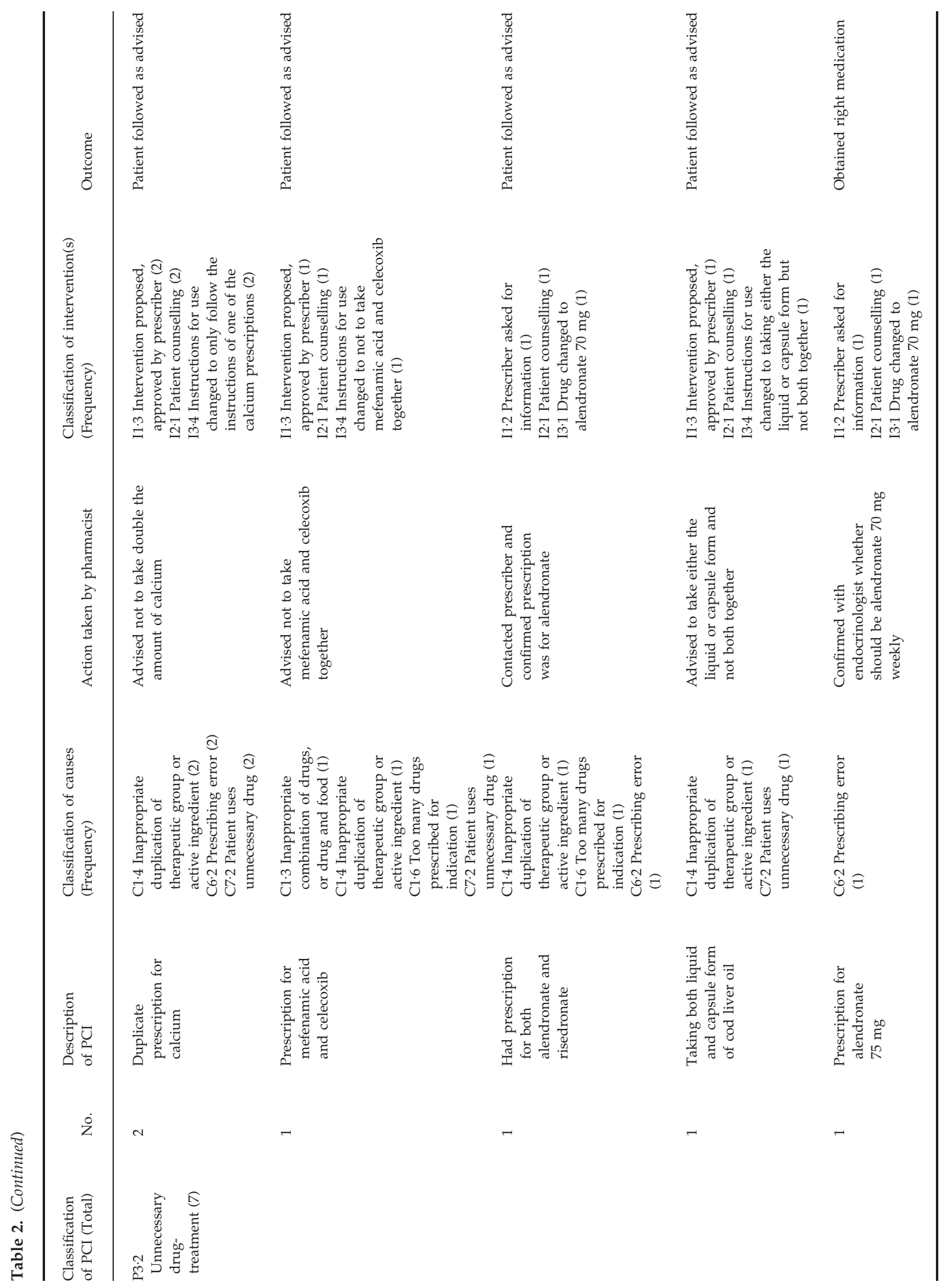




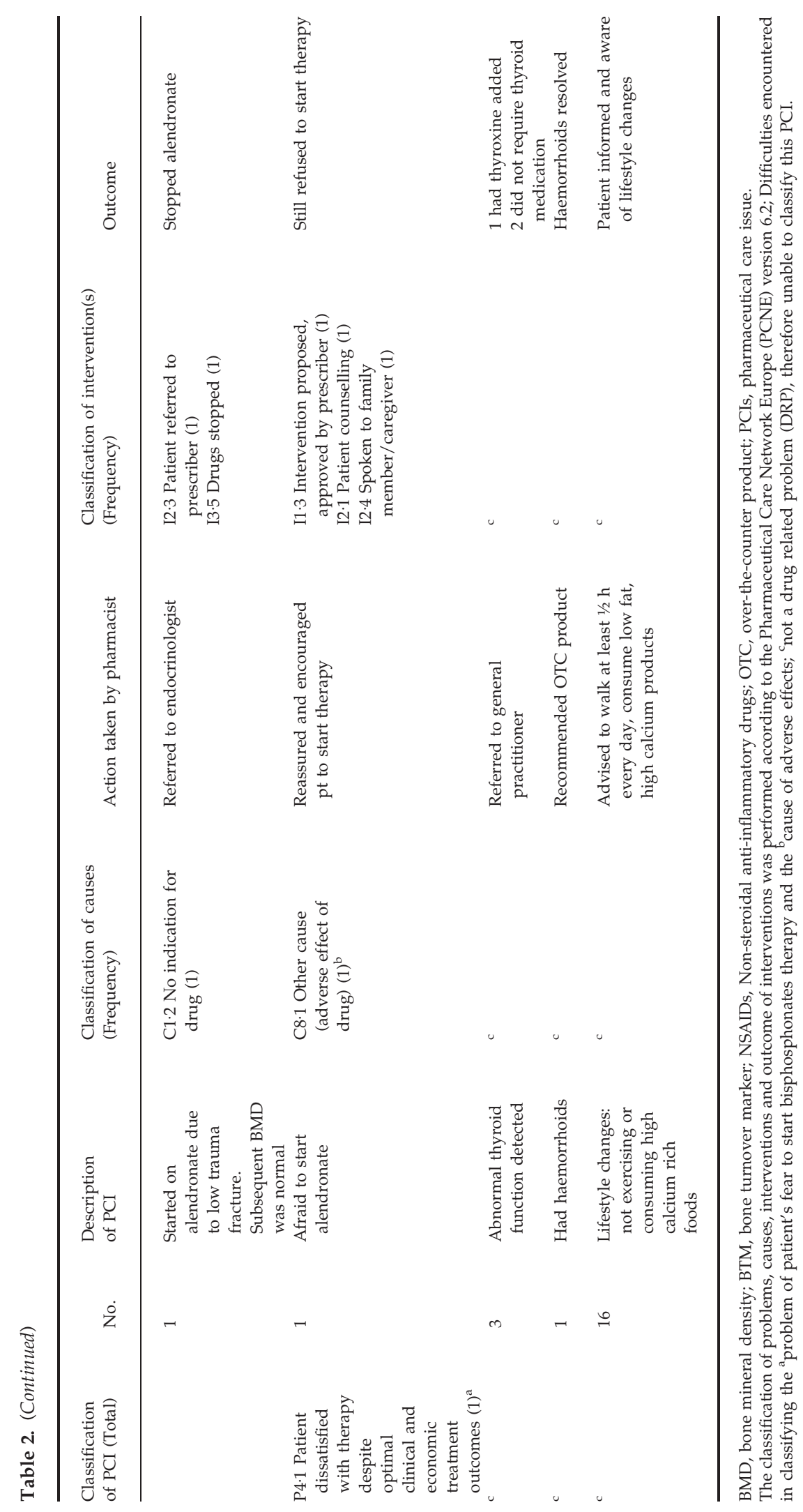




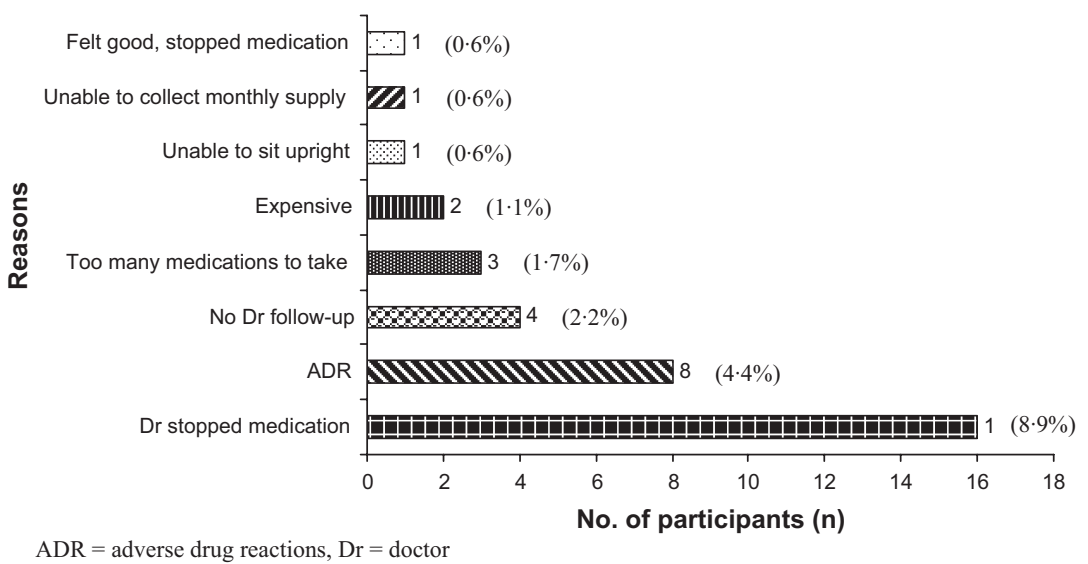

Fig. 1. Reasons for discontinuation of osteoporosis medications.

$\mathrm{ADR}=$ adverse drug reactions, $\mathrm{Dr}=$ doctor

no follow-up appointments scheduled, 2 (1.0\%) could not afford to buy osteoporosis medications and $1(0.5 \%)$ felt that it was inconvenient to see the doctor as the hospital was too far (one of the participants provided more than one reason). This is similar to previous studies which found that overall, $81.7 \%$ of appointments were kept after a period of 1 year, ${ }^{35}$ the median persistence for weekly alendronate was 3.8 years and the risk of discontinuing or changing therapy increased with age. ${ }^{36}$ This emphasizes the importance of continuity of care, especially for older patients and those who are still experiencing problems with their treatment.

A repeat BMD scan is usually required after 2 years of osteoporosis therapy. However, $53(26.8 \%)$ participants did not have their BMD scan performed because 15 (9.1\%) did not want to, $13(6 \cdot 6 \%)$ had no clinic follow-up, $10(5 \cdot 1 \%)$ could not be contacted and hence the reasons were not known, $10(5 \cdot 1 \%)$ did not have a BMD scan order written, two (1.0\%) had undergone an operation, two (1.0\%) passed away, whereas one $(0.5 \%)$ could not afford to pay for the BMD scan. Participants were also interested to know whether there was any improvement in their BMD scan. A significant improvement in the lumbar-spine and femoral neck BMD from baseline was observed after 2 years, indicating the efficacy of bisphosphonates therapy. Pharmacists should educate patients on the importance of having a BMD scan every 2 years as well as help monitor patients and remind physicians to order a BMD scan when required.

A total of $33(18.4 \%)$ participants reported 36 events that occurred during the 2 years after the initiation of bisphosphonates. The most frequently reported event was a fall (63.9\%). Of the 23 participants who had a fall, nine (39.1\%) had a fracture with the fall. In addition, 25 (13.9\%) participants reported that they were hospitalized. The most common reasons for the hospital admission were infections [seven (3.9\%)], operation [six (3.3\%)], fractures [four (2.2\%)] and GI problems [four (2.2\%)] which included gastric pain, food poisoning and diverticular disease. The incidence of falls reported by the participants after 2 years $(4.5 \%)$ appeared consistent with that of another study (3.2\%) which compared the incidence of fracture with placebo $(6 \cdot 2 \%){ }^{37}$ This should encourage osteoporosis patients to be more persistent with their medications.

The main strength of this study is that it describes in detail the PCIs encountered by post-menopausal osteoporotic women prescribed once-weekly bisphosphonates, which has been validated by a physician and classified according to the PCNE classification (v6-2). These findings will enable pharmacists to focus on the common PCIs encountered by osteoporotic patients during their counselling session.

One of the limitations in this study is that the research pharmacist was the same pharmacist who provided the intervention. The researcher could have carried out actions which may have resulted in higher numbers of PCIs reported, as patients are more likely to discuss their problems in a clinical intervention setting. However, it was not possible to determine fracture outcomes as 2 years were not long enough to evaluate such longterm outcome. In addition, data were collected from participants who understand English only and hence may be a more educated group (shown by $70 \%$ with at least secondary education). Further studies could be conducted with emphasis on pharmacists counselling patients on the possible adverse effects of medications, and actions to be taken over a longer period of time to detect fracture outcomes.

\section{WHAT IS NEW AND CONCLUSION}

The main PCIs identified were related to the use of bisphosphonates and its adverse effects. The study showed that the presence of a clinical pharmacist has enabled patients to voice their medication-related issues and to allow appropriate recommendations and actions to be taken to resolve these issues. However, further studies on the benefits of having a clinical pharmacist in the osteoporosis clinic will enable more real clinical practice issues to be determined.

\section{CONFLICT OF INTEREST}

The authors declare that they have no conflict of interest.

\section{ACKNOWLEDGEMENTS}

This project was funded by the Postgraduate Research Fund P0110/2006B and the Endocrine Research fund, University of Malaya.

We also like to extend our appreciation to Professor Dr Awang Bulgiba (Department of Social and Preventive Medicine, University of Malaya) for his advice on statistical procedures. Last but not least, we would like to thank all the research participants for their involvement in this study. 


\section{REFERENCES}

1. Kanis J, Melton LJ. The diagnosis of osteoporosis. J Bone Miner Res 1994;9:1137-1141.

2. International Osteoporosis Foundation. About osteoporosis (for health professionals): basic bone biology. International Osteoporosis Foundation; 2008. Available at: http://www.iofbonehealth.org/healthprofessionals/about-osteoporosis.html. (accessed 14 March 2008).

3. Davidson M. Pharmacotherapeutics for osteoporosis prevention and treatment. I Midwifery Womens Health 2003;48:39-54.

4. Melton LJ 3rd. The prevalence of osteoporosis: gender and racial comparison. Calcif Tissue Int 2001;69:179-181.

5. Lau EMC, Lee JK, Suriwongpaisal P, Saw SM, Das De S, Khir A, Sambrook P. The incidence of hip fracture in four Asian countries: the Asian Osteoporosis Study (AOS). Osteoporos Int 2001;12:239-243.

6. Hepler CD, Strand LM. Opportunities and responsibilities in pharmaceutical care. Am J Hosp Pharm 1990;47:533-543.

7. Pols HAP, Felsenberg D, Hanley DA et al. Multinational, placebo-controlled, randomized trial of the effects of alendronate on bone density and fracture risk in postmenopausal women with low bone mass: results of the FOSIT study. Osteoporos Int 1999;9:461-468.

8. Blau EM, Gordon SJ, Benton LK, Weiss TW, Chen YT. Establishment of an innovative clinic for postmenopausal women at high risk for fragility fracture. Drug Benefit Trends 2003;15:41-46.

9. Cerulli J, Zeolla MM. Impact and feasibility of a community pharmacy bone mineral density screening and education program. J Am Pharm Assoc 2004;44:161-167.

10. Cuddihy MT, Amadio PC, Gabriel SE, Pankratz VS, Kurland RL, Melton LJ. A prospective clinical practice intervention to improve osteoporosis management following distal forearm fracture. Osteoporos Int 2004;15:695-700.

11. Goode JV, Swiger K, Bluml BM. Regional osteoporosis screening, referral, and monitoring program in community pharmacies: findings from project ImPACT: osteoporosis. J Am Pharm Assoc 2004;44:152-160.

12. Gray M, Rajaei-Dahkordi Z, Ewan M, Wysocki R. Investigating the potential contribution of community pharmacists in identifying, understanding and meeting the bone health needs of patients in collaboration with GPs. Int J Pharm Pract, 2002;10(Suppl.):R34.

13. MacLaughlin EJ, MacLaughlin AA, Snella KA, Winston TS, Fike DS, Raehl CR. Osteoporosis screening and education in com- munity pharmacies using a team approach Pharmacotherapy 2005;25:379-386.

14. Newman ED, Hanus P. Improved bone health behavior using community pharmacists as educators - the geisinger health system community pharmacist osteoporosis education program. Dis Mana Heal Outcomes 2001;9:329-335.

15. Jaglal SB, Hawker G, Bansod V et al. A demonstration project of a multi-component educational intervention to improve integrated post-fracture osteoporosis care in five rural communities in Ontario, Canada. Osteoporos Int 2009;20:265-274.

16. Briot K, Ravaud P, Dargent-Molina P, Zylberman M, Liu-Leage S, Roux C. Persistence with teriparatide in postmenopausal osteoporosis; impact of a patient education and follow-up program: the French experience. Osteoporos Int 2008;20:625-630.

17. Kuo I, Ong C, Simmons L, Bliuc D, Eisman JA, Center JR. Successful direct intervention for osteoporosis in patients with minimal trauma fractures. Osteoporos Int 2007;18:1633-1639.

18. Curry LC, Hogstel MO, Davis GC, Frable PJ. Population based osteoporosis education for older women. Public Health Nurs 2002;19:460-469.

19. Lai P, Chua SS, Chew YY, Chan SP. Effects of pharmaceutical care on adherence and persistence to bisphosphonates in postmenopausal osteoporotic women. J Clin Pharm Ther, 2011;36:557-567.

20. Lai P, Chua SS, Chan SP. Does the knowledge of osteoporosis in postmenopausal osteoporotic women improve with pharmacist intervention? 8th Malaysian Pharmaceutical Society Pharmacy Scientific Conference, 77 Abstract PPP6, 2008.

21. Lai P, Chua SS, Chan SP. The impact of pharmaceutical care on the quality of life of postmenopausal osteoporotic women prescribed bisphosphonates. Mala J Pharm 2009;1:Abstract PPCP-21.

22. Dupont WD, Plummer WD. PS: power and sample size calculation. 2004. Available at: http://biostat.mc.vanderbilt.edu/ twiki/bin/view/Main/PowerSampleSize, (updated 12 June; accessed 30 June 2007).

23. Schousboe JT, DeBold RC, Kuno LS, Weiss TW, Chen YT, Abbott TA. Education and phone follow-up in postmenopausal women at risk for osteoporosis Effects on calcium intake, exercise frequency, and medication use. Dis Manage Heal Outcomes 2005;13:395-404.

24. Abramson JH, Abramson ZH. Survey methods in community medicine. ed. 5th edn. Edinburgh: Churchill Livingstone, 1999.

25. Foppe van Mil JW. Classification for drug related problems (version 6.2). Pharma- ceutical Care Network Europe Foundation, 2010 (updated 14 January 2010; cited 201029 Nov).

26. Sewerynek E, Dabrowska K, Skowronska-Jozwiak E, Zygmunt A, Lewinski A. Compliance with alendronate 10 treatment in elderly women with postmenopausal osteoporosis. Endocrinol Pol 2009;60:76-81.

27. Clowes JA, Peel NFA, Eastell R. The impact of monitoring on adherence and persistence with antiresorptive treatment for postmenopausal osteoporosis: a randomized controlled trial. J Clin Endocrinol Metab 2004;89:1117-1123.

28. Papaioannou A, Kennedy C, Dolovich L, Lau E, Adachi J. Patient adherence to osteoporosis medications: problems, consequences and management strategies [Review]. Drugs Aging 2007;24:37-55.

29. Lappe J. Nonadherence to osteoporosis medications: a critical problem. Clin Rev Bone Mineral Metabol 2006;4:25-32.

30. Weycker D, Macarios D, Edelsberg J, Oster G. Compliance with drug therapy for postmenopausal osteoporosis. Osteoporos Int 2006;17:1645-1652.

31. Quek D. Countering patients' irrational fear of side effects. 1997 Available at: http:// www.vadscorner.com/editorial0597.html (accessed 15 September).

32. Wilson S, Wahler R, Brown J, Doloresco F, Monte SV. Impact of pharmacist intervention on clinical outcomes in the palliative care setting. Am J Hosp Palliat Care 2011;28:316-320.

33. Von Muenster S, Carter B, Weber C, Ernst M, Milchak J, Steffensmeier J, Xu Y. Description of pharmacist interventions during physician-pharmacist co-management of hypertension. Pharm World Sci 2008;30:128-135.

34. Roberts MS, Stokes JA, King MA et al. Outcomes of a randomized controlled trial of a clinical pharmacy intervention in 52 nursing homes. $\mathrm{Br} J$ Clin Pharmacol 2001;51:257-265.

35. Murray MJ, LeBlanc $\mathrm{CH}$. Clinic follow-up from the emergency department: do patients show up? Ann Emerg Med 1996;27:56-58.

36. Roerholt C, Eiken P, Abrahamsen B. Initiation of anti-osteoporotic therapy in patients with recent fractures: a nationwide analysis of prescription rates and persistence. Osteoporos Int 2009;20:299-307.

37. Liberman UA, Weiss SR, Broll J, Minne HW, Quan H, Bell NH. Effect of oral alendronate on bone mineral density and the incidence of fractures in postmenopausal osteoporosis: the Alendronate Phase III Osteoporosis Treatment Study group. N Engl J Med 1995;333:1437-1443. 